Bull. Austral. Math. Soc.

$47 \mathrm{~A} 16,47 \mathrm{~A} 11,46 \mathrm{~A} 04,46 \mathrm{~A} 16$

VoL. $70(2004) \quad[45-54]$

\title{
ON HYPERCYCLICITY AND SUPERCYCLICITY CRITERIA
}

\author{
Teresa Bermúdez, Antonio Bonilla and Alfredo Peris
}

\begin{abstract}
We show that the Hypercyclicity Criterion coincides with other existing hypercyclicity criteria and prove that a wide class of hypercyclic operators satisfy the Criterion. The results obtained extend or improve earlier work of several authors. We also unify the different versions of the Supercyclicity Criterion and show that operators with dense generalised kernel and dense range are supercyclic.
\end{abstract}

\section{Introduction and Preliminaries}

Let $E$ be a separable infinite dimensional $\mathcal{F}$-space (that is, a complete and metrisable topological vector space) over the scalar field $\mathbb{K}=\mathbb{R}$ or $\mathbb{C}$ and denote by $L(E)$ the space of all continuous linear operators on $E$. An operator $T \in L(E)$ is said to be cyclic if there is a vector $x \in E$, called cyclic vector, such that the (forward) orbit $\operatorname{Orb}(T, x):=\left\{x, T x, T^{2} x, \ldots\right\}$, has dense linear span (that is, $\overline{\operatorname{span}(\operatorname{Orb}(T, x))}=E$ ). The operator is supercyclic if there exists a vector $x \in E$ (called supercyclic for $T$ ) such that the set of scalar multiples of the orbit is dense. And $T$ is hypercyclic if there exists a vector $x \in E$ (called hypercyclic for $T$ ) such that the orbit itself is dense. A vector $x \in E$ is periodic for $T$ if there is $n \in \mathbb{N}$ such that $T^{n} x=x$, and $x$ is called eventually periodic if there exist $n, m \in \mathbb{N}$ with $n>m$ such that $T^{n} x=T^{m} x$. In the case that we have a sequence of operators $\left\{T_{n}\right\}_{n \in \mathbb{N}} \subset L(E)$ and $x \in E$ such that its (forward) orbit satisfies $\overline{\left\{T_{n} x: n \in \mathbb{N}\right\}}=E$, the sequence $\left\{T_{n}\right\}_{n \in \mathbb{N}}$ is said to be universal. By backward orbit of a vector $x \in E$ under $\left\{T_{n}\right\}_{n \in \mathbb{N}}$, if it exists, we mean any sequence $\left\{x_{0}, x_{1}, x_{2}, \ldots\right\}$ in $E$ such that $x_{0}=x$ and $T_{n} x_{n}=x$ for all $n \in \mathbb{N}$.

Examples of universality appeared in 1914 see [22], but the first example of a hypercyclic operator on a Fréchet space (that is, locally convex and $\mathcal{F}$-space) was given by

Received 14th October, 2003

The authors' would like to thank Luis Bernal and Karl Grosse-Erdmann who informed us about the coincidence of some of their results with ours in Section 2, gave us a copy of their paper [5], and finally made some interesting comments about our article. We are specially indebted to José Bonet who carefully revised the manuscript and improved the presentation.

The first author was partially supported by Consejería de Educación del Gobierno de Canarias PI 2002023 (Spain) and by MCYT and FEDER, BFM 2003-07139. The second author was supported by MCYT and FEDER, BFM2002-02098. The third author was supported by MCYT and FEDER, BFM 2001-2670 and AVCIT Grupo 03/050.

Copyright Clearance Centre, Inc. Serial-fee code: 0004-9727/04 \$A2.00+0.00. 
Birkhoff [8] in 1929. Indeed, he proved that the translation operator $T_{a} f(z)=f(z+a)$ with $a \neq 0$ is hypercyclic on the space $\mathcal{H}(\mathbb{C})$ of entire functions on the complex plane, endowed with the compact-open topology. MacLane [20], in 1952, proved the hypercyclicity of the differentiation operator on $\mathcal{H}(\mathbb{C})$. However, the first example of a hypercyclic operator on a Banach space was given by Rolewicz [24] who showed that scalar multiples of the unilateral backward shift are hypercyclic on $\ell^{p}(1 \leqslant p<\infty)$ for any scalar with modulus greater than 1 . In fact, the first sufficient condition for hypercyclicity (the Hypercyclicity Criterion) discovered independently by Kitai [18] and Gethner and Shapiro [12], was motivated by Rolewicz's example. Finally Bès [6] gave the most general version of the Hypercyclicity Criterion (see also [7]). For some classes of operators hypercyclicity is equivalent to the Hypercyclicity Criterion. In particular, Salas gave a characterisation of hypercyclic weighted shifts in terms of the weights [25]. Chaotic operators (that is, hypercyclic operators with a dense set of periodic points) and hypercyclic operators with dense generalised kernel also satisfy the Hypercyclicity Criterion [7, Proposition 2.14 and 2.11]. It is not known whether there exists a hypercyclic operator that does not satisfy the Hypercyclicity Criterion. For motivation and background about hypercyclicity and universality we refer the reader to the survey of Grosse-Erdmann [14].

The definition and first properties of supercyclic operators were introduced by Hilden and Wallen [17]. They proved that all unilateral backward weighted shifts on a Hilbert space have a supercyclic vector. The study of supercyclic operators has experienced a great of development in recent years. Salas gave a characterisation of supercyclic bilateral backward weighted shifts via the Supercyclicity Criterion (that is, a sufficient condition for supercyclicity) [26]. Montes and Salas [21] refined the Supercyclicity Criterion and proved that it is equivalent to the former one given by Salas. Recently, Feldman, Miller and Miller [11, Theorems 5.1 and 5.2] gave new supercyclicity criteria.

We shall be concerned with the study of universality, hypercyclicity and supercyclicity criteria. This problem has received the attention of several authors (for example, see $[7,11,13,14,21,26]$ and, more recently, Bernal and Grosse-Erdmann [5]).

Universality criteria for operators on $\mathcal{F}$-spaces are analysed in Section 2 . Actually, we show that the sufficient conditions for universality given by Grosse-Erdmann [14, Theorem 2] are equivalent to the Universality Criterion for commuting sequences of operators. As an application we obtain new assumptions, expressed in terms of the existence of many eigenvalues and eigenvectors, which are sufficient for an operator to satisfy the Hypercyclicity Criterion. For instance, we show that every chaotic operator in a $\mathcal{F}$-space satisfies the Hypercyclicity Criterion, generalising some results of [7]. Moreover, we prove that every hypercyclic operator, which is the transpose of a multiplication operator in certain Banach spaces of analytic functions, satisfies the Criterion. This improves some known results.

Finally in Section 3, we unify all the supercyclicity criteria using some ideas of [7]. 
We also characterise the supercyclicity of operators with dense generalised kernel.

After writing a first version of this paper, it was shown in [23] that the original Gethner-Shapiro's hypercyclicity criterion is equivalent to Bès criterion. This result, together with the characterisations presented in this paper, closes the study of the equivalence of hypercyclicity criteria.

\section{UNIVERSALITY AND HYPERCYCLICITY CRITERIA}

This section is devoted to the study of equivalences of (a priori) different hypercyclicity and universality criteria. We shall also show that large classes of universal and hypercyclic operators satisfy these criteria.

We shall assume from now on in this section that $E$ is a separable $\mathcal{F}$-space and $\left\{T_{n}\right\}_{n \in \mathbb{N}} \subset L(E)$ is a sequence of operators.

Properties (A) and (B) below are sufficient conditions for the existence of a dense $G_{\delta}$-subset of $E$ consisting of universal vectors for the sequence $\left\{T_{n}\right\}_{n \in \mathbf{N}}$. Property (A) was given by Bès and Peris in [7, Remark 2.6(3)], and it is referred to as the "Universality Criterion". Property (B) is due to Grosse-Erdmann in [14, Theorem 2].

THEOREM 2.1. Let $\left\{T_{n}\right\}_{n \in \mathbb{N}}$ be a commuting sequence of operators. Then the following are equivalent:

(A) (Universality Criterion) There exist $X_{0}$ and $Y_{0}$ dense subsets of $E,\left\{n_{k}\right\}_{k \in \mathbb{N}} \subset \mathbb{N}$ strictly increasing, and mappings $S_{n_{k}}: Y_{0} \rightarrow E$ so that

(i) $T_{n_{k}} \rightarrow 0$ pointwise on $X_{0}$,

(ii) $S_{n_{k}} \rightarrow 0$ pointwise on $Y_{0}$, and

(iii) $T_{n_{k}} S_{n_{k}} \rightarrow I d_{Y_{0}}$ pointwise on $Y_{0}$.

(B) There exist dense subsets $X_{0}$ and $Y_{0}$ of $E,\left\{n_{k}\right\}_{k \in \mathbb{N}} \subset \mathbb{N}$ strictly increasing, and mappings $S_{n_{k}}: Y_{0} \rightarrow E$ such that

1. for every $x \in X_{0}$, there exists a subsequence $\left\{m_{j}\right\}$ of $\left\{n_{k}\right\}$ such that $T_{m_{j}} x$ $\rightarrow 0$,

2. for every $y \in Y_{0},\left\{S_{n_{k}} y\right\}$ converges,

3. for every $y \in Y_{0},\left(T_{n_{k}} \circ S_{n_{k}}\right) y \rightarrow y$.

Proof: It is clear that if $\left\{T_{n}\right\}_{n \in N}$ satisfies (A), then it also satisfies (B). Conversely, suppose that conditions 1,2 and 3 of $(B)$ are satisfied. Since there is a universal vector $z \in E$, we can redefine $X_{0}:=\left\{z, T_{n_{1}} z, T_{n_{2}} z, \ldots\right\}$, which is dense. If we select $\left\{r_{k}\right\} \subset\left\{n_{k}\right\}$ such that $T_{r_{k}} z \rightarrow 0$, then we also have that $T_{r_{k}} x \rightarrow 0$ for every $x \in X_{0}$ by commutativity of the sequence of operators. The sequence $\left\{T_{r_{k}}\right\}_{k \in \mathbb{N}}$ also satisfies the conditions of (B), therefore it is (densely) universal. Then we can find a subsequence of $\left\{r_{k}\right\}$, which for convenience is assumed to coincide with $\left\{r_{k}\right\}$, and a sequence of vectors $\left\{w_{k}\right\} \subset E$ such that $w_{k} \rightarrow 0$ and $T_{r_{k}} w_{k} \rightarrow z$. If we redefine $Y_{0}:=X_{0}$ and $S_{r_{k}}: Y_{0} \rightarrow E$ by 
$S_{r_{k}}\left(T_{n} z\right):=T_{n} w_{k}$, the commutativity of the operators easily gives conditions (ii) and (iii) of the Universality Criterion.

REMARK 2.2. Grosse-Erdmann noticed in [14, Remark 2] that, in the condition (B) above, if all the limits in 2 are zero, then one may weaken 1 to mere convergence. Furthermore, the quantifier 'there exists a subsequence $\left\{m_{j}\right\}$ ' can be shifted from 1 to 2 or 3 . These versions are also equivalent to the Universality Criterion under the hypotheses of our theorem.

An interesting consequence of this result is that the Universality Criterion is satisfied by for those universal sequences of operators that admit a dense subset of vectors with precompact forward orbit (respectively, backward orbit).

COROLLARY 2.3. If $\left\{T_{n}\right\}_{n \in \mathbf{N}} \subset L(E)$ is a universal sequence of commuting operators such that, either

(1) there is a dense set of vectors whose orbit is precompact, or

(2) there is a dense set of vectors that admit a precompact backward orbit, then $\left\{T_{n}\right\}_{n \in \mathbb{N}}$ satisfies the Universality Criterion.

Proof: (1) or (2) imply the conditions of (B) in Theorem 2.1. Indeed, in case (1), we define $X_{0}$ as the set of vectors whose orbit is precompact. Now, given a universal vector $z$ for $\left\{T_{n}\right\}$, we find an increasing sequence $\left\{n_{k}\right\}$ and a sequence of vectors $\left\{w_{k}\right\}$ $C E$ such that $w_{k} \rightarrow 0$ and $T_{n_{k}} w_{k} \rightarrow z$. We define $Y_{0}:=\left\{z, T_{1} z, T_{2} z, \ldots\right\}$ and $S_{n_{k}}$ : $Y_{0} \rightarrow E$ by $S_{n_{k}}\left(T_{n} z\right):=T_{n} w_{k}$. Conditions 1,2 and 3 of $(\mathrm{B})$, in the form mentioned in Remark 2.2, are then satisfied. In case (2), if $z$ is a universal vector for $\left\{T_{n}\right\}$, we define $X_{0}$ $:=\left\{z, T_{1} z, T_{2} z, \ldots\right\}$ and find an increasing sequence $\left\{n_{k}\right\}$ such that $T_{n_{k}} z \rightarrow 0$. We set $Y_{0}$ to be the dense set of vectors which admit precompact backward orbit. For each $y \in Y_{0}$, we fix a precompact backward orbit $\left\{y_{0}, y_{1}, \ldots\right\}$ and define $S_{n_{k}} y:=y_{n_{k}}, k \in \mathbb{N}$. Properties 1, 2 and 3 of (B) are satisfied as formulated in Remark 2.2.

The fact that (1) implies the Universality Criterion was also proved independently by Bernal and Grosse-Erdmann [5, Theorem 4.1] weakening the commutativity of the sequence (what they define as "weakly commuting") and using a different argument.

We shall apply the previous results to hypercyclicity. They remain valid in this context since the set of iterates of a single operator form a commuting sequence. The following definition, due to Bès [6] (see also [7]), the most general version of the Hypercyclicity Criterion. It inspired the definition of the Universality Criterion mentioned before (see [7, Remark 2.6(3)]). The hypotheses of the Hypercyclicity Criterion are equivalent to the hypercyclicity of $T \oplus T$, and also to the existence of an increasing sequence $\left\{n_{k}\right\}$ such that the sequence $\left\{T^{m_{j}}\right\}$ is hypercyclic for every subsequence $\left\{m_{j}\right\}$ of $\left\{n_{k}\right\}$, that is, to be hereditarily hypercyclic (see [7, Theorem 2.3]).

Definition 2.4: (Hypercyclicity Criterion) A continuous linear operator $T$ on a separable $\mathcal{F}$-space $E$ satisfies the Hypercyclicity Criterion if there exist an increasing 
sequence of positive integers $\left\{n_{k}\right\}_{k \in \mathbb{N}}$ and

1. a dense subset $X_{0} \subset E$ such that $T^{n_{k}} x \longrightarrow 0$ for every $x \in X_{0}$, and

2. a dense subset $Y_{0} \subset E$ and a sequence of mappings $S_{n_{k}}: Y_{0} \longrightarrow E$ such that

(a) $S_{n_{k}} y \longrightarrow 0$ for every $y \in Y_{0}$,

(b) $T^{n_{k}} S_{n_{k}} y \longrightarrow y$ for every $y \in Y_{0}$.

The following corollary covers a wide class of hypercyclic operators. It appeals to the existence of many eigenvectors and eigenvalues (See also [1], [2, Theorem 7], [3, Theorem $3],[4$, Theorem 2.2], [11] and [16]).

Corollary 2.5. Let $T$ be a hypercyclic operator on an $\mathcal{F}$-space $E$. If either the linear span of $\bigcup_{\substack{n>m \\|\lambda| \leqslant 1}} \operatorname{Ker}\left(T^{n}-\lambda T^{m}\right)$ is dense or the linear span of $\bigcup_{\substack{n \in \mathbb{N} \\|\lambda| \geqslant 1}} \operatorname{Ker}\left(\lambda-T^{n}\right)$ is dense, then $T$ satisfies the Hypercyclicity Criterion.

In particular, if $T$ is a hypercyclic operator with a dense subset of eventually periodic points, then $T$ satisfies the Hypercyclicity Criterion.

PROOF: If $X_{0}:=\operatorname{span} \bigcup_{\substack{n>m \\|\lambda| \leqslant 1}} \operatorname{Ker}\left(T^{n}-\lambda T^{m}\right)$ is dense, then every $x \in X_{0}$ satisfies $\operatorname{Orb}(T, x) \subset\left\{\alpha T^{i} x:|\alpha| \leqslant 1, i=1, \ldots n\right\}$ for some $n$, which is precompact. In the second case $Y_{0}:=\operatorname{span} \bigcup_{\substack{n \in \mathbb{N} \\|\lambda| \geqslant 1}} \operatorname{Ker}\left(\lambda-T^{n}\right)$ is dense, and then every $y \in Y_{0}$ admits a backward orbit which is contained in $\left\{\alpha T^{i} y:|\alpha| \leqslant 1, i=1, \ldots n\right\}$ for some $n$, which is precompact. The conclusion follows from Corollary 2.3 .

In particular, the set

$X:=\{$ eventually periodic points of $T\}=\bigcup_{n>m} \operatorname{Ker}\left(T^{n}-T^{m}\right)$.

REMARKS 2.6. (1) Using the corollary we can unify and generalise two results of [7]. Concretely, if $T$ has a dense generalised kernel, that is $\bigcup_{n \in \mathbb{N}} \operatorname{Ker}\left(T^{n}\right)$ is dense, and $T$ is hypercyclic, then $T$ satisfies the Hypercyclicity Criterion [7, Proposition 2.11] and, if $T$ is chaotic in the sense of Devaney [10] then it satisfies the Hypercyclicity Criterion [7, Proposition 2.14]. Moreover, we should mention that [7, Proposition 2.14] was proved just for Fréchet spaces (that is, locally convex $\mathcal{F}$-spaces). See also [5, Corollary 4.3].

(2) Feldman, Miller and Miller [11, Theorem 3.2] proved that if $T$ is an operator on a complex separable Banach space $X$ such that the glocal spectral subspaces $\mathcal{X}_{T}(\mathbb{D})$ and $\mathcal{X}_{T}(\mathbb{C} \backslash \overline{\mathbb{D}})$ are dense, then $T$ satisfies the Hypercyclicity Criterion. We note that if $T$ is hypercyclic and either $\mathcal{X}_{T}(\mathbb{D})$ or $\mathcal{X}_{T}(\mathbb{C} \backslash \overline{\mathbb{D}})$ is dense, then $T$ satisfies the Hypercyclicity Criterion. In the first case $X$ has a dense subset of vectors whose orbits are convergent to zero and in the second case $X$ has a dense subset of vectors that admit a backward orbit converging to zero. For definitions on local spectral theory see [19].

Let $\Omega$ be a domain (connected, open set) in $\mathbb{C}^{n}$ and $X$ a reflexive Banach space of analytic functions on $\Omega$ such that the point evaluations are continuous on $X$. If 
$\phi: \Omega \longrightarrow \mathbb{C}$ is a bounded analytic function such that $\phi f \in X$, for all $f \in X$, then define the multiplication operator $M_{\phi}: X \longrightarrow X$ by $M_{\phi} f:=\phi f$, which is a bounded operator.

Godefroy and Shapiro proved that, for Hilbert spaces $X$, if $\phi$ is a non-constant function such that $\phi(\Omega) \cap \partial \mathbb{D} \neq \emptyset$, then the adjoint operator $M_{\phi}^{*}$ satisfies the Hypercyclicity Criterion [13, Theorem 4.5]. Bonet, Domanski and Lindström [9, Section 4] showed that the above condition is equivalent to the fact that the transpose multiplier $M_{\phi}^{\prime}$ is chaotic on $X^{\prime}=\left(H_{v}^{0}\right)^{\prime}$, the dual of the Banach space of analytic functions $f$ on the disc such that the radial weight $v$ and the product $f v$ tend to zero at the boundary. Our goal is to show that every hypercyclic operator which is the transpose of a multiplication operator on $X$ satisfies the Criterion. This also covers the adjoint multipliers when $X$ is a Hilbert space. Under the specified assumptions on $X$ we have the following result.

COROLlary 2.7. If $\phi$ is a non-constant function and the transpose $M_{\phi}^{\prime}$ of $M_{\phi}$ is hypercyclic, then $M_{\phi}^{\prime}$ satisfies the Hypercyclicity Criterion.

Proof: We have the following three possibilities:

(1) $\phi(\Omega) \cap \partial \mathbb{D} \neq \emptyset$,

(2) $\phi(\Omega) \subset \mathbb{D}$ or

(3) $\phi(\Omega) \subset \mathbb{C} \backslash \overline{\mathbb{D}}$.

Case (1) is shown to imply the Hypercyclicity Criterion in [13, Theorem 4.5]. In cases (2) and (3), by the boundedness of the point evaluations, for each $z \in \Omega$ there is $K_{z} \in X^{\prime}$ so that $f(z)=\left\langle f, K_{z}\right\rangle$ for all $f \in X$. Clearly, $K_{z} \in \operatorname{Ker}\left(M_{\phi}^{\prime}-\phi(z)\right)$. Hence, for each $z \in \Omega$, we have that $\phi(z)$ is an eigenvalue of $M_{\phi}^{\prime}$ and, moreover, by [13, Proposition 2.4] we have that either span $\bigcup_{|\lambda| \leqslant 1} \operatorname{Ker}(\lambda-T)$ is dense or span $\bigcup_{|\lambda| \geqslant 1} \operatorname{Ker}(\lambda-T)$ is dense. Using Corollary 2.5 we obtain that $M_{\phi}^{\prime}$ satisfies the Hypercyclicity Criterion.

REMARK 2.8. It is natural to ask about the existence of hypercyclic operators $M_{\phi}^{\prime}$ such that $\phi(\Omega)$ does not intersect the unit circle. A precise example is $\phi(z):=z$ on the disc, as a multiplier of the Dirichlet space $\mathcal{D}$ of the unit disc, which consists of all functions $f$ holomorphic on $\mathbb{D}$ for which the derivative $f^{\prime}$ is square integrable with respect to Lebesgue area measure. In this case $M_{\phi}^{\prime}$ is the backward shift on the Bergman space $A^{2}$, the space of holomorphic functions on $\mathbb{D}$ that are square integrable with respect to Lebesgue area measure, which is hypercyclic [12], but $\phi(\Omega)$ does not intersect the unit circle.

\section{SUPERCYCLICITY CRITERIA}

The purpose of this section is to unify the apparently different versions of supercyclicity criteria that have appeared in the literature. The "unifying link" is the supercyclicity of the direct sum of the operator with itself.

The definitions of universality and supercyclicity are closely related: if $T: E$ $\longrightarrow E$ is supercyclic, then there are an increasing sequence $\left\{n_{k}\right\}_{k \in \mathbb{N}} \subset \mathbb{N}$ of integers 
and $\left\{\lambda_{n_{k}}\right\}_{k \in \mathbb{N}} \subset \mathbb{K}$, such that the sequence $\left\{\lambda_{n_{k}} T^{n_{k}}\right\}_{k \in \mathbb{N}}$ is universal. Just note that, if $\left\{\lambda T^{n} x: n \in \mathbb{N}, \lambda \in \mathbb{K}\right\}$ is dense in $E$, then $\left\{\lambda T^{n} x: n \geqslant m, \lambda \in \mathbb{K}\right\}$ is also dense, for each $m \in \mathbb{N}$. We shall use this fact repeatedly throughout this section.

In order to prove that all supercyclicity criteria are equivalent to the supercyclicity of $T \oplus T$, we adapt some ideas of Bès and Peris [7] in this context. The following lemma introduces what we shall call the Supercyclicity Criterion, which is a slight modification of the one given by Montes and Salas [21, Theorem 2.2] (see also the last paragraph of [21, Final Comments (1)]). This result was also proved by Bès (personal communication).

LEMMA 3.1. Let $T$ be an operator on a separable $\mathcal{F}$-space. Then $T \oplus T$ is supercyclic if and only if $T$ satisfies the hypotheses of the following Supercyclicity Criterion:

There exists an increasing sequence of positive integers $\left\{n_{k}\right\}_{k \in \mathbb{N}} \subset \mathbb{N}$ and a sequence $\left\{\lambda_{n_{k}}\right\}_{k \in \mathbf{N}} \subset \mathbb{K} \backslash\{0\}$ for which there are

1. A dense subset $X_{0} \subset E$ such that $\lambda_{n_{k}} T^{n_{k}} x \rightarrow 0$ for every $x \in X_{0}$.

2. A dense subset $Y_{0} \subset E$ and a sequence of mappings $S_{n_{k}}: Y_{0} \longrightarrow X$ such that

(a) $\left(1 / \lambda_{n_{k}}\right) S_{n_{k}} y \longrightarrow 0$ for every $y \in Y_{0}$.

(b) $T^{n_{k}} S_{n_{k}} y \longrightarrow y$ for every $y \in Y_{0}$.

Proof: If $T$ satisfies the Supercyclicity Criterion, then by [7, Remark 2.6] we have that there exists $\left(n_{k}\right)$ such that $T_{n_{k}} \oplus T_{n_{k}}$ is universal, where $T_{n_{k}}:=\lambda_{n_{k}} T^{n_{k}}$ for certain sequence $\left\{\lambda_{n_{k}}\right\}_{k \in \mathbb{N}} \subset \mathbb{K} \backslash\{0\}$. But this is equivalent to the supercyclicity of $T \oplus T$. For the reverse implication we also use [7, Remark 2.6] to conclude that $\left\{\lambda_{n_{k}} T^{n_{k}}\right\}_{k \in N}$ satisfies the Universality Criterion for some $\left(n_{k}\right)$ and $\left\{\lambda_{n_{k}}\right\}_{k \in \mathbb{N}} \subset \mathbb{K} \backslash\{0\}$. That is, $T$ satisfies the Supercyclicity Criterion.

Supercyclic operators have been mainly studied in the context of (complex) Banach spaces. Therefore it was natural to state the criteria in the Banach setting having in mind the concrete applications of these criteria. We shall show the equivalence of two supercyclicity criteria given by Feldman, Miller and Miller in [11] to the Supercyclicity Criterion.

THEOREM 3.2. Let $X$ be a separable Banach space and $T \in L(X)$. Then the following are equivalent:

(a) T satisfies the Supercyclicity Criterion.

(b) (Outer Supercyclicity Criterion, [11, Theorem 5.1]) There exist an increasing sequence of positive integers $\left\{n_{k}\right\}_{k \in \mathbb{N}} \subset \mathbb{N}$, a dense linear subspace $Y_{0}$ and, for each $y \in Y_{0}$, a dense linear subspace $X_{y}$ such that:

1. There exists a sequence of maps $S_{n_{k}}: Y_{0} \rightarrow X$ such that $T^{n_{k}} S_{n_{k}} y \rightarrow y$ for all $y \in Y_{0}$, and

2. $\left\|T^{n_{k}} x\right\|\left\|S_{n_{k}} y\right\| \rightarrow 0$ for every $y \in Y_{0}$ and $x \in X_{y}$.

(c) (Inner Supercyclicity Criterion, [11, Theorem 5.2]) There exist an increasing sequence of positive integers $\left\{n_{k}\right\}_{k \in \mathbb{N}} \subset \mathbb{N}$, a dense linear subspace $Y_{0}$ and, for each 
$y \in Y_{0}$, a dense linear subspace $X_{y}$ such that:

1. There exists a sequence of maps $S_{y, n_{k}}: X_{y} \rightarrow X$ such that $T^{n_{k}} S_{y, n_{k}} x \rightarrow x$ for all $x \in X_{y}$, and

2. $\left\|T^{n_{k}} y\right\|\left\|S_{y, n_{k}} x\right\| \rightarrow 0$ for every $y \in Y_{0}$ and $x \in X_{y}$.

Proof: It is obvious that any operator satisfying the Supercyclicity Criterion also satisfies the criteria of (b) and (c). We are done if we show the supercyclicity of $T \oplus T$ for any operator $T$ satisfying (b) or (c). We shall prove this for the Outer Criterion (b) since the other case is analogous.

Let $U_{i}, V_{i} \subset X$ non-empty open sets with $i=1,2$. We have to show that there exist $\lambda \in \mathbb{K}$ and $m \in \mathbb{N}$ such that

$$
\lambda T^{m} U_{i} \cap V_{i} \neq \emptyset, \text { for } i=1,2 .
$$

If we fix $y_{i} \in Y_{0} \cap V_{i}$, then there exists $X_{y_{i}} \subset X$ dense such that

$$
\lim _{k \rightarrow \infty}\left\|T^{n_{k}} x\right\|\left\|S_{n_{k}} y_{i}\right\|=0, \quad \forall x \in X_{y_{i}}, \text { for } i=1,2
$$

Passing to a subsequence, if necessary, we suppose without loss of generality that $\left\|S_{n_{k}} y_{1}\right\| \leqslant\left\|S_{n_{k}} y_{2}\right\|$ for all $k \in \mathbb{N}$. Thus

$$
\lim _{k \rightarrow \infty}\left\|T^{n_{k}} x\right\|\left\|S_{n_{k}} y_{1}\right\| \leqslant \lim _{k \rightarrow \infty}\left\|T^{n_{k}} x\right\|\left\|S_{n_{k}} y_{2}\right\|=0, \quad \forall x \in X_{y_{2}}
$$

Given $x_{i} \in X_{y_{2}} \cap U_{i}$, we select $m \in \mathbb{N}$ "big enough" and $\varepsilon>0$ such that

$$
x_{i}+\varepsilon B_{X} \subset U_{i}, \quad T^{m} S_{m} y_{i}+\varepsilon B_{X} \subset V_{i} \text {, and }\left\|T^{m} x_{i}\right\|\left\|S_{m} y_{j}\right\|<\varepsilon^{2},
$$

for $i, j=1,2$. We then define $\lambda:=\left\|S_{m} y_{2}\right\| / \varepsilon$. This implies that $\left\|\lambda T^{m} r_{i}\right\|<\varepsilon, i=1,2$ and, therefore,

$$
\lambda T^{m}\left(x_{i}+\frac{1}{\lambda} S_{m} y_{i}\right)=\lambda T^{m} x_{i}+T^{m} S_{m} y_{i} \in V_{i} \cap \lambda T^{m}\left(U_{i}\right), \text { for } i=1,2
$$

Hilden and Wallen [17] proved that unilateral backward weighted shifts are supercyclic on $l_{p}$. Salas [26, Corollary 2.8] improved this result and obtained that any operator $T$ on a separable $\mathcal{F}$-space whose generalised kernel $Y:=\bigcup_{n=1}^{\infty} \operatorname{Ker} T^{n}$ is dense and such that $\left.T\right|_{Y}$ admits a (possibly discontinuous and non-linear) right inverse, satisfies the Supercyclicity Criterion. The following result characterises, in a clean way, the supercyclicity of operators with dense generalised kernel.

Corollary 3.3. Let $E$ be a separable $\mathcal{F}$-space and $T \in L(E)$ with dense generalised kernel. Then the following conditions are equivalent:

1. $T$ has a dense range,

2. $T$ is supercyclic, 


\section{T satisfies the Supercyclicity Criterion.}

Proof: Obviously (3) $\Rightarrow(2) \Rightarrow(1)$. The implication $(1) \Rightarrow(3)$ goes as follows: Given $U$ and $V$ non-empty open sets of $E \oplus E$, we first pick $\left(x_{1}, x_{2}\right) \in U$ such that $T^{n} x_{1}$ $=T^{n} x_{2}=0$ for some $n \in \mathbb{N}$. Then we select $\left(y_{1}, y_{2}\right) \in E \oplus E$ such that $\left(T^{n} y_{1}, T^{n} y_{2}\right) \in V$, by the density of the range of $T$. For suitable $\lambda>0$ we have

$$
\frac{1}{\lambda}\left(y_{1}, y_{2}\right)+\left(x_{1}, x_{2}\right) \in U
$$

and also

$$
\lambda\left(T^{n} \oplus T^{n}\right)\left(\frac{1}{\lambda}\left(y_{1}, y_{2}\right)+\left(x_{1}, x_{2}\right)\right)=\left(T^{n} y_{1}, T^{n} y_{2}\right) \in V .
$$

Therefore $T \oplus T$ is supercyclic and we get (3) from Lemma 3.1.

We finally observe that our argument shows that operators $T$ with dense generalised kernel and dense range satisfy the Supercyclicity Criterion with respect to the sequence of all positive integers. In other words, the sequence $\left\{T^{n_{k}}\right\}_{k \in \mathbb{N}}$ is supercyclic for every $\left(n_{k}\right)$ strictly increasing [7, Remark 2.6(3)]. Interesting operators of this type are the ones constructed by Herzog [15] on any infinite dimensional separable Banach space.

\section{REFERENCES}

[1] T. Bermúdez and V. Miller, 'On operators $T$ such that $f(T)$ is hypercyclic', Integral Equation Operator Theory 37 (2000), 332-340.

[2] L. Bernal, 'Hypercyclic sequences of differential and antidifferential operators', J. Approx. Theory 96 (2000), 323-337.

[3] L. Bernal, 'Densely hereditarily hypercyclic sequences and large hypercyclic manifolds', Proc. Amer. Math. Soc. 127 (1999), 3279-3285.

[4] L. Bernal, J.A. Prado-Tendero, 'Sequences of differential operators: exponentials, hypercyclicity and equicontinuity', Ann. Polon. Math. 77 (2001), 169-187.

[5] L. Bernal and K.G. Grosse-Erdmann, 'The hypercyclicity criterion for sequences of operators', Studia Math. 157 (2003), 17-32.

[6] J. Bès, Three problems on hypercyclic operators, Ph.D. thesis (Kent State University, 1998).

[7] J. Bès and A. Peris, 'Hereditarily hypercyclic operators', J. Funct. Anal. 167 (1999), 94-112.

[8] G.D. Birkhoff, 'Démonstration d'un théorème élémentaire sur les fonctions entières', $C$. R. Acad. Sci. Paris 189 (1929), 473-475.

[9] J. Bonet, P. Domański and M. Lindström, 'Pointwise multiplication operators on weighted Banach spaces of analytic functions', Studia Math. 137 (1999), 177-194.

[10] R.L. Devaney, An introduction to chaotic dynamical systems, (2nd ed.) (Addison-Wesley, Reading, MA, 1989).

[11] N. Feldman, V. Miller and L. Miller, 'Hypercyclic and supercyclic cohyponormal operators', Acta Sci. Math. 68 (2002), 303-328.

[12] R.M. Gethner and J. Shapiro, 'Universal vectors for operators on space of holomorphic functions', Proc. Amer. Math. Soc. 100 (1987), 281-288. 
[13] G. Godefroy and J. Shapiro, 'Operators with dense invariant cyclic vectors manifolds', J. Funct. Anal. 98 (1991), 229-269.

[14] K.-G. Grosse-Erdmann, 'Universal families and hypercyclic operators', Bull. Amer. Math Soc. 36 (1999), 345-381.

[15] G. Herzog, 'On linear having supercyclic operators', Studia Math. 103 (1992), 295-298.

[16] G. Herzog and C. Schmoeger, 'On operators $T$ such that $f(T)$ is hypercyclic', Studia Math. 108 (1994), 209-216.

[17] H. Hilden and L. Wallen, 'Some cyclic and non-cyclic vectors of certain operators', Indiana Univ. Math. J. 23 (1974), 557-565.

[18] C. Kitai, Invariant closed sets for linear operators, Thesis (Univ. Toronto, 1982).

[19] K. B. Laursen and M. M. Neumann, Introduction to local spectral theory, London Math. Soc. Monographs New Series 20 (Oxford University Press, New York, 2000).

[20] G.R. MacLane, 'Sequences of derivatives and normal families', J. Analyse Math. 2 (1952), $72-87$.

[21] A. Montes-Rodríguez and H. Salas, 'Supercyclic subspaces: spectral theory and weighted shifts', Adv. Math. 163 (2001), 74-134.

[22] J. Pál, 'Zwei Kleine Bemerkungen', Tôhoku Math. J. 6 (1914/15), 42-43.

[23] A. Peris, 'Hypercyclicity criteria and the Mittag-Leffler theorem', Bull. Soc. Roy. Sci. Liège 70 (2001), 365-371.

[24] S. Rolewicz, 'On orbits of elements', Stud. Math. 33 (1969), 17-22.

[25] H. Salas, 'Hypercyclic weighted shifts', Trans. Amer. Math. Soc. 347 (993-1004).

[26] H. Salas, 'Supercyclicity and weighted shifts', Studia Math. 135 (1999), 55-74.

Departamento de Análisis Matemático

Universidad de La Laguna

38271 La Laguna (Tenerife)

Spain

e-mail: tbermude@ull.es

E.T.S. Arquitectura

Departament de Matemàtica Aplicada

Universitat Politècnica de València

E-46022 València

Spain

e-mail: aperis@mat.upv.es

\author{
Departamento de Análisis Matemático \\ Universidad de La Laguna \\ 38271 La Laguna (Tenerife) \\ Spain \\ e-mail: abonilla@ull.es
}

